\title{
Biophysical investigation of curcumin based nanocomposite for wound dressing application
}

\author{
Nagwa A. Kamel ${ }^{1 *}$, Ahmed A. F. Soliman ${ }^{2}$, Nehad N. Rozik ${ }^{3}$, Salwa L. Abd-Elmessieh ${ }^{1}$ \\ ${ }^{1}$ Microwave Physics and Dielectrics Department, Physics Division, National Research Centre, Dokki, Cairo, Egypt. \\ ${ }^{2}$ Pharmacognosy Department, Pharmaceutical and Drug Industries Research Division, National Research Centre, Dokki, Cairo, Egypt. \\ ${ }^{3}$ Polymers and Pigments Department, Chemical Industries Research Division, National Research Centre, Dokki, Cairo, Egypt.
}

\section{ARTICLE INFO \\ Article history: \\ Received on: 15/11/2017 \\ Accepted on: 06/04/2018 \\ Available online: 30/05/2018}

\section{Key words:}

dielectric, chitosan,

polyvinylalcohol,

nanocurcumin,

nanocomposite, wound

dressing.

\begin{abstract}
Curcumin (cur) loaded polyvinyl alcohol (PVA), chitosan (Cs) and PVA/Cs blend (50:50) were prepared with different concentrations of curcumin $(0,5,10$, and $15 \mathrm{wt} \%)$ using the solution casting technique and studied for wound dressing application. Physical characterization of the composites achieved through dielectric spectroscopy (DS), fourier transform infrared spectroscopy (FTIR), scanning electron microscope (SEM) and transmission electron microscope (TEM). The wound dressing characteristics were evaluated by the water vapor transmission rate (WVTR), antimicrobial studies and cell viability assay. The scanning electron microscope revealed good dispersion of the nanocurcumin in the matrix. The dielectric properties of all composites were studied through the measurements of the permittivity $\varepsilon^{\prime}$ and dielectric loss $\varepsilon^{\prime \prime}$. These results show that both values for PVA/cur nanocomposites greatly increase with increasing curcumin content which was found to be higher than those of the two individuals. On the opposite side, both $\varepsilon^{\prime}$ and $\varepsilon^{\prime \prime}$ decrease with increasing curcumin content in case of Cs/cur and PVA/Cs/cur nanocomposites. The obtained electrical conductivity for all composites lies in the order of $\left(10^{-9}-10^{-14} / \mathrm{S} \mathrm{cm}^{-1}\right)$. WVTR of the samples decreased by increasing the curcumin \%. Results of the cell viability assay confirmed that the addition of the curcumin has no toxic effect on the cells. PVA/cur nanocomposites displayed excellent antimicrobial activity.
\end{abstract}

\section{INTRODUCTION}

Functional biomaterials research has built on new drug delivery systems and enhanced scaffolds for regenerative medicine that is recently one of the most developing fields in the life sciences. Polyvinyl alcohol (PVA) and chitosan (Cs) are biopolymers that are widely used in this field due to their biocompatibility, nontoxicity, noncarcinogenicity, and their swelling properties (Baker et al., 2012; Dash et al., 2011).

Polymer blends are physical mixtures of two or more polymers. Usually, the properties of the blend are superior to the component homopolymers. Some studies on the polyvinyl

\footnotetext{
${ }^{*}$ Corresponding Author

Nagwa A. Kamel, Researcher, Microwave Physics \& Dielectrics Dep., Physics Research Division, National Research Centre, El Behoos St. Dokki 12622, Cairo, Egypt.

E-mail:Nagwakamel@gmail.com; Na.kamel@nrc.sci.eg
}

alcohol (PVA)/chitosan blends have been reported. Nakano et al. (2007) studied the mechanical and thermal properties of chitosan and polyvinyl alcohol (PVA). The characteristics of the blend films studied using DSC, X-ray, viscoelastic and positron annihilation techniques. The results recommended that the miscibility of chitosan and PVA could be confirmed by entanglement of the amorphous chain segments of chitosan and PVA.

The adhesion and growth of fibroblasts on the PVA and PVA/Cs membranes were investigated by Chuang et al. (1999). They concluded that PVA membrane can be modified by blending with chitosan. In addition, they observed that, for cell culture, the PVA/Cs blend was more favorable than the pure PVA.

Curcumin (diferuloylmethane, 1, 7-bis (4-hydroxy-3methoxyphenyl)-1, 6-heptadiene-3, 5-dione) is a polyphenolic compound which is a natural product obtained from an Asian spice called turmeric. Curcumin is a member of the ginger family. It is believed to have many biological activities such as antioxidant, 
anti-inflammatory, and even anticancer effect. Several studies suggest that it might reduce inflammation and pain symptoms resulted from osteoarthritis and rheumatoid arthritis (Weber et al., 2005; Shankar et al., 2012; Fan et al. 2013).

Curcumin has been revealed high cytotoxicity towards different cancer cells, but its instability and water-insolubility make its bioavailability extremely low and thus it usually displays low anticancer activity in in-vivo tests (Tang et al., 2010). Several studies have demonstrated the therapeutic effects of curcumin (Aggarwal and Kuzhuvelil, 2009; Duvoix et al., 2005; Phan et al., 2001).

Curcumin-loaded Poly (lactic acid) (PLA) membranes were studied to be used as a drug-eluting stent. The anticoagulation assay conducted through the platelet adhesion test, enlightening that the anticoagulation behavior of the membranes was higher than the pure PLA membrane and the anticoagulation improved by increasing curcumin percent (Chen et al., 2010).

The release of curcumin from PVA/curcumin composites and its effectiveness towards hepatocarcinoma were studied by El-Nashar et al. (2016). They concluded that the rate of release for curcumin depends on $\mathrm{pH}$ of the media and that (PVA/cur) composites have a hopeful result on human liver cancer cell $\mathrm{HEPG}_{2}$.

Novel hydrogel-silver nanoparticle-curcumin composites have been studied by Varaprasad et al. (2011) as wound dressing and antibacterial application. The results demonstrate that combining the natural compound curcumin enhances the antimicrobial effect and the composites have potential applications in the wound dressing or burn wounds.

PVA and Cs blend in different compositions with and without incorporation of curcumin have been prepared by Vidyalakshmi et al. (2004) and evaluated as drug delivery system. Mechanical properties of the membranes such as percentage elongation at break and tensile strength were evaluated.

The overall goal of this work is to study the result of loading curcumin with different concentrations $(0,5,10$, and 15 $\mathrm{wt} \%$ ) on the dielectric and biological properties of PVA, chitosan and PVA/Cs blend (50:50) to be used for medical applications, especially for wound healing.

\section{MATERIALS AND METHODS}

\section{Materials}

Chitosan: poly (D-glucosamine), beta-(1, 4)-2-Amino2-deoxy-D-glucose $\left(\mathrm{C}_{6} \mathrm{H}_{11} \mathrm{NO}_{4}\right)_{\mathrm{n}}$ (degree of deacetylation $(\mathrm{DD})=$ 75-92\%). Houston, Texas, USA.

Polyvinylalcohol: $\left(\mathrm{C}_{2} \mathrm{H}_{4} \mathrm{O}\right)_{\mathrm{n}}$ supplied from Qualikems fine chemicals Pvt.Ltd., New Delhi. Commercial Curcumin purchased from markets.

Gram-Negative Bacteria: (Escherichia coli (ATCC25922), Salmonella enterica (ATCC 25566), Klebsiella pneumonia (ATCC 10031) and Psedomonas aeruginosa (ATCC 27853). Gram Positive Bacteria: (Bacillus subtilis (NRRL-B4219), Staphylococcus aureus (ATCC 6538) and Streptococcus pyogenes (ATCC19615). Yeasts:(Candidaalbicans (ATCC10231).

HFB4 (Human normal melanocyte) cell line was purchased from Vacsera (Giza, Egypt).

\section{Methods}

\section{Synthesis of nano curcumin}

Curcumin solution was prepared by dissolving curcumin powder in ethanol. Under ultrasonication condition, this solution was added to boiling water in drop-wise mode. The solution was sonicated for about $30 \mathrm{~min}$. After which, the mixture was stirred at $800 \mathrm{rpm}$ for about $20 \mathrm{~min}$ till we obtained the orange colored precipitate. Afterward, the supernatant was removed and the obtained pellet was used for the study.

\section{Preparation of curcumin nanocomposites}

The PVA was dissolved in aqueous solution by using tetrahydrofuran (THF), heated in a water bath and stirred for 30 min. chitosan dissolved in $2 \%$ aqueous acetic acid solution at room temperature with stirring to prepare chitosan solution.

Curcumin-loaded PVA, CS, and PVA/Cs blend (50:50) nanocomposites were prepared. Curcumin of various concentrations, i.e., $0,5,10$, and $15 \mathrm{wt} \%$ were dissolved in ethanol with PVA, $\mathrm{C}_{\mathrm{S}}$, and PVA/Cs (50/50). The resulting solution was stirred carefully few hours until a homogenous yellow color solution is obtained. Finally, the solution was poured into glass Petri dish and left to evaporate the solvent at room temperature overnight.

\section{Transmission electron microscope (TEM)}

The particle size of the nano-curcumin powder was determined using Transmission electron microscope (TEM) model: JEM-HR 2100 and accelerating voltage $200 \mathrm{kV}$ (Japan).

\section{Scanning electron microscope (SEM)}

The homogeneity of the samples and distribution of the curcumin powder in the polymer matrix was investigated by Scanning electron microscope, SEM/EDX, Philips XL30 (Japan).

\section{Fourier transform infrared (FTIR)}

The infrared spectrum was recorded by a JASCO FT/ IR 300 E Fourier transform infrared (FTIR) Spectrometer (Tokyo, Japan).

\section{Dielectric measurements}

An LCR meter type AG-411 B (Ando electric, Japan) was used to measure the dielectric properties in the frequency range $100 \mathrm{~Hz}$ up to $100 \mathrm{kHz}$ at room temperature $\left(25^{\circ} \mathrm{C}\right)$. The bridge directly measured the capacitance $\mathrm{C}$, loss tangent $\tan \delta$ and ac resistance $\left(\mathrm{R}_{\mathrm{ac}}\right)$ and from those parameters, the permittivity $\dot{\varepsilon}$, dielectric loss $\varepsilon^{\prime \prime}$, and $\mathrm{R}_{\mathrm{dc}}$ were calculated. A guard ring capacitor type NFM/5T Wiss Tech. Werkstatten (WTW) GMBH Germany was used as a measuring cell. Standard materials used to calibrate the cell and the experimental error in $\varepsilon$ and $\varepsilon^{\prime \prime}$ were found to be $\pm 3 \%$ and $\pm 5 \%$, respectively.

\section{Water vapor transmission rate (WVTR)}

The water vapor transmission rate (WVTR) was measured according to the monograph of the European pharmacopeia (Razzak and Darwis, 2001). Glass bottles were filled with $20 \mathrm{ml}$ water. Circular shape samples with thickness $0.75 \mathrm{~mm}$ adhered to 
the bottles as a cap as seen in Figure 1. The bottles were weighed $\left(\mathrm{W}_{\mathrm{i}}\right)$ and kept at $37^{\circ} \mathrm{C}$ in an incubator for 24 hours. After 24 hours the bottles are weighed $\left(\mathrm{W}_{\mathrm{f}}\right)$ and the WVTR was calculated from:

$$
\text { WVTR }(\mathrm{g} / \mathrm{m} 2 / \mathrm{h})=\frac{\text { Wi-Wf }}{244} \times 10^{6} \quad 1
$$

where $\mathrm{A}$ is the surface area of the circular sample in $\left(\mathrm{mm}^{2}\right)$.

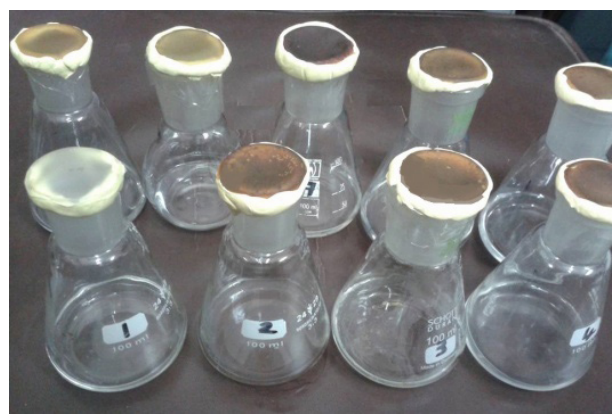

Fig. 1: Experiment for WVTR

\section{Cell viability assay}

HFB4 (Human normal melanocyte) was preserved in Dulbecco's Modified Eagle's Medium (DMEM). The medium was supplemented with $10 \%$ fetal bovine serum and incubated at $37^{\circ} \mathrm{C}$ in $5 \% \mathrm{CO}_{2}$ and $95 \%$ humidity. The cell line was sub-cultured using trypsin $0.15 \%$. After $24 \mathrm{~h}$ of seeding 10000 cells per well (in 96 well plates), the medium was changed to serum-free medium containing a final concentration of the tested samples of $100 \mu \mathrm{g} / \mathrm{ml}$ in triplicates. The cell was treated for $48 \mathrm{~h} .100 \mu \mathrm{g} / \mathrm{ml}$ doxorubicin was used as positive control and $0.5 \%$ DMSO was used as negative control. Cell viability was determined using the MTT (3-(4, 5-dimethylthiazol-2-yl)-2, 5-diphenyltetrazolium bromide) assay (Mosmann, 1983). The equation used for calculation of percentage cytotoxicity:

$$
\text { percentage cytotoxicity }=(\operatorname{av}(x) /(\operatorname{av}(N C))) \times 100,
$$

where Av: average, X: absorbance of the sample well measured at $595 \mathrm{~nm}$ with reference $690 \mathrm{~nm}, \mathrm{NC}$ : absorbance of negative control measured at $595 \mathrm{~nm}$ with reference 690.

\section{Antimicrobial assay}

Composites loaded with 0 and $10 \mathrm{wt} \%$ cur were subjected to microbiology study using a disk diffusion methodology (BSAC Disk Diffusion Method for Antimicrobial Susceptibility Testing, Version 4, 2005). Isosensitest agar (Oxoid, Basingstoke, UK). The sample code as follow:

\begin{tabular}{|c|c|c|c|c|c|}
\hline 1 & 2 & 3 & 4 & 5 & 6 \\
\hline PVA & PVA/cur & Cs & $\mathrm{Cs} /$ cur & PVA/Cs & PVA/Cs/cur \\
\hline
\end{tabular}

The tested samples placed onto the inoculated plates and incubated overnight at $37^{\circ} \mathrm{C}$. The diameters of the inhibition zones formed around the disks were measured. Levofloxacin antibiotic discs with potency $(5 \mathrm{mcg})$ were used as a standard antibiotic (Rahman et al., 2007; Andrews, 2002).

\section{RESULTS AND DISCUSSION}

\section{Transmission electron microscope (TEM)}

Figure 2 shows the TEM image of nano-curcumin particles. The micrograph shows spherical ultrafine particles in the nano grade with diameter ranges from 2-4 $\mathrm{nm}$.

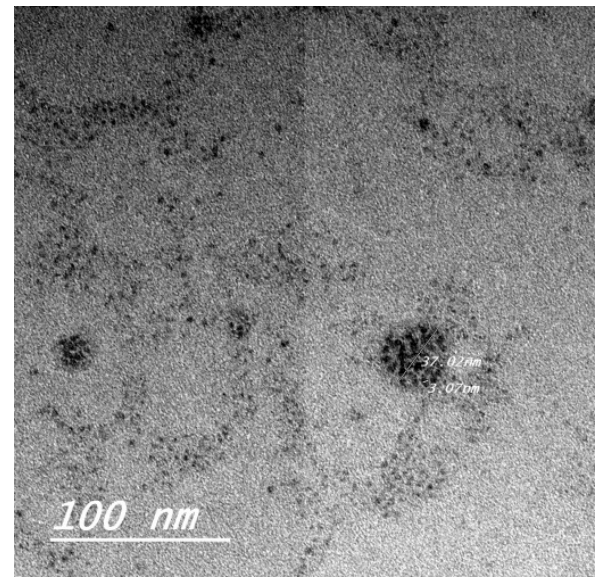

Fig. 2: TEM of Nano-Curcumin.

\section{Scanning electron microscope (SEM)}

The morphology of the PVA, Cs and PVA/Cs blend and their cur nanocomposites with different concentrations were studied using SEM technique and only PVA/Cs blend and 10\% cur loaded samples presented in Figure 3 for the sake of brevity.

The surface morphology of pure PVA, Cs and PVA/Cs samples are found to be smooth and homogenous. The addition of nanocurcumin particles causes changes in the surface morphology of such polymers. The curcumin nanoparticles are homogeneously distributed in the polymer matrix with some aggregations at the higher concentration.

\section{Fourier transform infrared spectroscopy (FTIR)}

To investigate the interaction between nano curcumin with PVA, Cs and PVA/Cs blend, FTIR spectra were measured and recorded in the spectral range from $400-4000 \mathrm{~cm}^{-1}$ and represented in Figures 4-8 and the band assignments of pure PVA and chitosan in Table 1. The characteristic bands of Cs and PVA in this study are in agreement with that reported before in the literature (Kamel et al., 2017; Wang et al., 2004; El-Hefian et al., 2010).

The FTIR spectrum of the PVA/Cs blend (Fig. 4) is different from that of the pure homopolymers. By comparing the spectra of pure Cs with the blend, the absorption peak at $3520 \mathrm{~cm}^{-1}$ characteristics for $-\mathrm{OH}$ and $-\mathrm{NH}$ stretching vibrations shifted to a $3620 \mathrm{~cm}^{-1}$ in the blend. The band at $1595 \mathrm{~cm}^{-1}$ in the pure chitosan spectrum characteristic for Amide II, N-H bending vibration and the symmetric- $\mathrm{NH}_{3}^{+}$deformation is shifted to $1582 \mathrm{~cm}^{-1}$ with an increase in the intensity. These results reflect the chemical interaction between the two polymers. A similar observation was mentioned in previous work on PVA/Cs studies (El-Hefian et al., 2010).

The chemical formula for curcumin is $\mathrm{C}_{21} \mathrm{H}_{20} \mathrm{O}_{6}$. The chemical structure and FTIR spectrum are given in Scheme 1 and Figure 5, respectively. 
Table 1: Main IR band assignments of PVA and Cs.

\begin{tabular}{|c|c|c|c|}
\hline \multicolumn{2}{|c|}{ Main IR band assignments of PVA } & \multicolumn{2}{|r|}{ Main IR band assignments of Cs } \\
\hline Wave number $\mathrm{cm}^{-1}$ & Band assignments & Wave number $\mathrm{cm}^{-1}$ & Band assignments \\
\hline 3600 & OH stretching vibrations & 3503 & O-H Stretching vibration and amine N-H bonds \\
\hline 2934 & $\mathrm{CH}$ stretching vibration of alkyl groups & 2898 & $\mathrm{C}-\mathrm{H}$ vibration \\
\hline 1747 & $\mathrm{C}=\mathrm{O}$ stretching vibration of the carbonyl group & 1660 & Amide I and antisymmetric- $\mathrm{NH}_{3}{ }^{+}$deformation \\
\hline 1640 & acetyl $\mathrm{C}=\mathrm{O}$ group & 1585 & $\begin{array}{l}\text { Amide II, N-H bending vibration and the symmetric- } \mathrm{NH}_{3}^{+} \\
\text {deformation }\end{array}$ \\
\hline 1470 & The corresponding bending of $\mathrm{CH}_{2}$ vibrations & 1434 & $\mathrm{C}-\mathrm{H}$ and $\mathrm{O}-\mathrm{H}$ deformation vibrations \\
\hline 1370 & The corresponding wagging of $\mathrm{CH}_{2}$ vibrations & 1277 & $\mathrm{C}-\mathrm{OH}$ stretching \\
\hline 1146 & The C-C and C-O stretching vibration band & $\begin{array}{l}1169 \\
1115\end{array}$ & $\begin{array}{l}\mathrm{CO} \text { stretching and } \mathrm{OH} \text { deformation vibrations of the primary and } \\
\text { secondary alcohol groups in the chitosan }\end{array}$ \\
\hline 1046 & C-O stretching & & \\
\hline
\end{tabular}
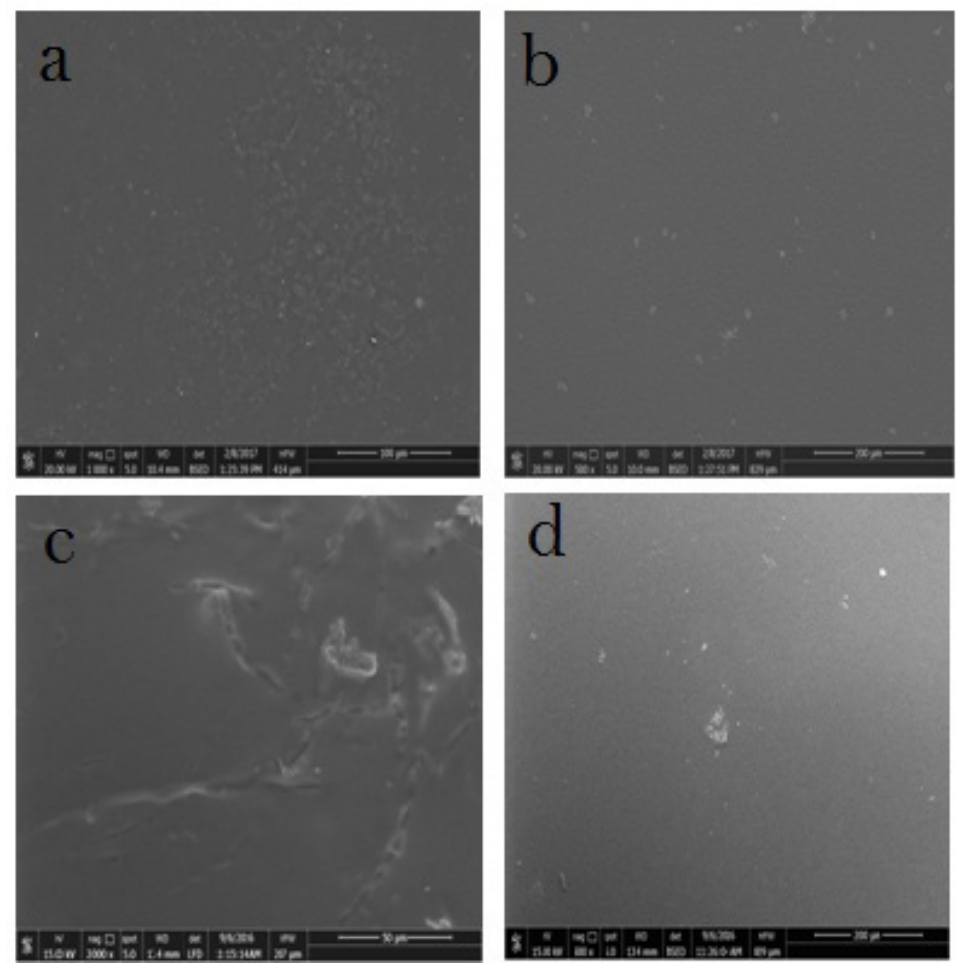

Fig. 3: SEM of (a) PVA/cur 10\% (b) Cs/cur 10\% (c) PVA/Cs and (d) PVA/Cs/cur $10 \%$.

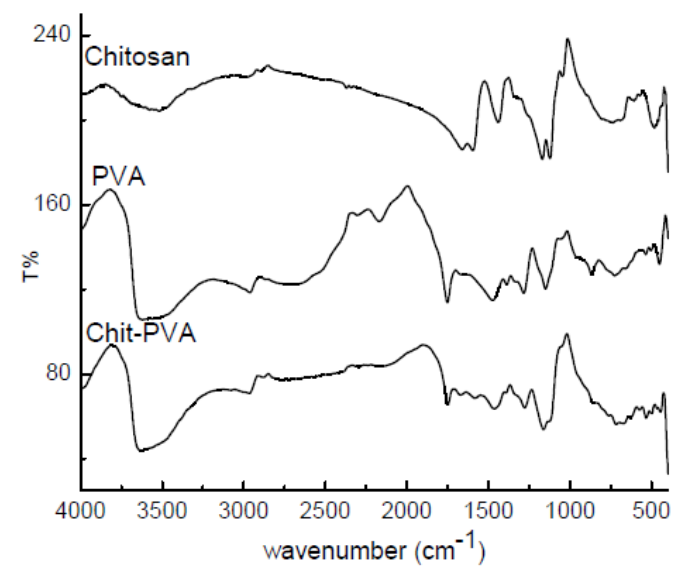

Fig. 4: FTIR spectra for PVA, Cs, and PVA/Cs blend.
As illustrated in scheme 1, the curcumin structure has 3 chemical entities: 2 aromatic rings containing o-methoxy phenolic groups, linked with 7 carbon linker consisting of an $\alpha, \beta$ unsaturated $\beta$-diketone moiety (Priyadarsini and Kavirayani 2014; Zhao et al., 2015).

Figure 5 shows the FTIR spectrum of curcumin. From the figure, a broadband appears at $3417 \mathrm{~cm}^{-1}$ attributed to the phenolic O-H stretching, 2 sharp peaks at 2920 and $2852.2 \mathrm{~cm}^{-1}$ attributed to $\mathrm{CH}$ Stretching, $1706 \mathrm{~cm}^{-1}$ for $\mathrm{C}=\mathrm{O}, 1641 \mathrm{~cm}^{-1}$ for $\mathrm{C}=\mathrm{C}$ stretching, 1464 for $v(\mathrm{OH}), 1105$ for $\mathrm{C}-\mathrm{O}-\mathrm{C}$ stretching modes, 723 for cis $\mathrm{CH}$ vibration of aromatic ring.

Figure 6 represents the FTIR spectra of PVA/cur nanocomposites with different concentrations of curcumin $(0,5$, 10 , and $15 \mathrm{wt} \%)$. From the figure, it can be noticed that a new band appeared at $1660 \mathrm{~cm}^{-1}$ as a result of curcumin (stretching vibration peak of the carbon-carbon double bond in curcumin and 
the intensity of this band increases by increasing the curcumin concentration. It is noticed also increasing in the intensities of the bands at 2963. 2173, 1773, 1471, and $1145 \mathrm{~cm}^{-1}$ after addition of curcumin. From the results of the FTIR, it appears that PVA and curcumin present in the nanocomposite without chemical interaction between them.<smiles>COc1cc(/C=C/C(=O)CC(=O)/C=C/c2ccc(O)c(OC)c2)ccc1O</smiles>

Scheme 1: Structure of curcumin.

For Cs/cur nanocomposites (Figure 7), the $1662 \mathrm{~cm}^{-1}$ band vibration characteristic for the Amide I and antisymmetric- $\mathrm{NH}_{3}^{+}$ of chitosan was shifted to $1672 \mathrm{~cm}^{-1}$. The band at $2898 \mathrm{~cm}^{-1}$ is increased up to $10 \%$ cur. Increased intensity of 1595,1440 and $1172 \mathrm{~cm}^{-1}$ bands also noticed with increasing cur $\%$. The resulted spectra may indicate the presence of interaction between the positively charged amino group of chitosan and negatively charged hydroxide group of curcumin (Das et al., 2010).

Figure 8 shows the FTIR spectra of PVA/Cs/cur nanocomposites with different concentrations of curcumin. The curcumin loaded PVA/Cs blend (Figure 8) has shown all the characteristic bands of the pure blend with a slight shift of the bands at 1160,1582 , and $1671 \mathrm{~cm}^{-1}$. The disappearance of the band at $1582 \mathrm{~cm}^{-1}$ from the spectrum of 5 and $10 \%$ cur loaded chit/PVA blend. The stretching vibration at $3627 \mathrm{~cm}^{-1}$ corresponding to $\mathrm{OH} /$ $\mathrm{NH}_{2}$ groups has shifted to $3649 \mathrm{~cm}^{-1}$, indicating that the curcumin has been bonded to the functional groups of the chitosan and PVA.

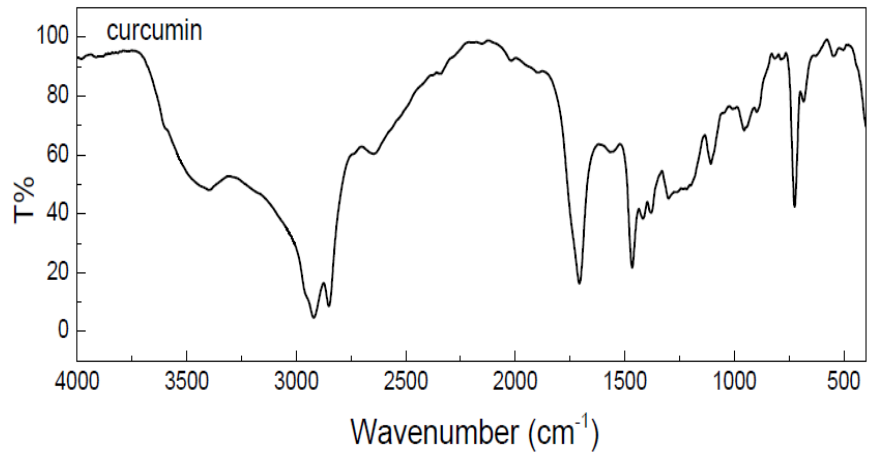

Fig. 5: FTIR spectrum of curcumin.

\section{Dielectric properties}

The permittivity $\varepsilon^{\prime}$ and dielectric loss $\varepsilon^{\prime \prime}$ for PVA, $\mathrm{Cs}$, and PVA/Cs blend loaded with different concentrations of curcumin versus the applied frequency $\mathrm{f}$, at room temperature $25^{\circ} \mathrm{C}$ are illustrated graphically in Figure 9. It is notable that the permittivity $\varepsilon^{\prime}$ for the whole samples shows a relatively high value at low-frequency range, decreased with increasing frequency and almost remained independent of the applied external field at the high-frequency range. It may be accomplished that; at a lower frequency, the dipoles within the system reorient themselves to retort to the applied electrical field. Whereas at a higher frequency, the decrease of permittivity values was attributed to the lag of molecules towards the applied electric field and this might be explained by the issue of dipoles orientation during this frequency (Srivastava et al., 2014). Moreover, it had been additionally observed that the values of $\varepsilon^{\prime}$ of PVA/cur nanocomposites greatly increase with increasing curcumin content which was found to be higher than those of the two individuals. This finding may be due to some sort of humidity of cur itself. On the opposite side, the permittivity decreases with increasing curcumin content in case of $\mathrm{CS} /$ cur and $\mathrm{PVA} / \mathrm{CS} /$ cur nanocomposites as a result of the fact that the value of $\varepsilon^{\prime}$ for cur is about 8.5 (Khan, 2014) while it is 6.9 for PVA and 33.3 for $\mathrm{Cs}$ at $\mathrm{f}=100 \mathrm{~Hz}$.

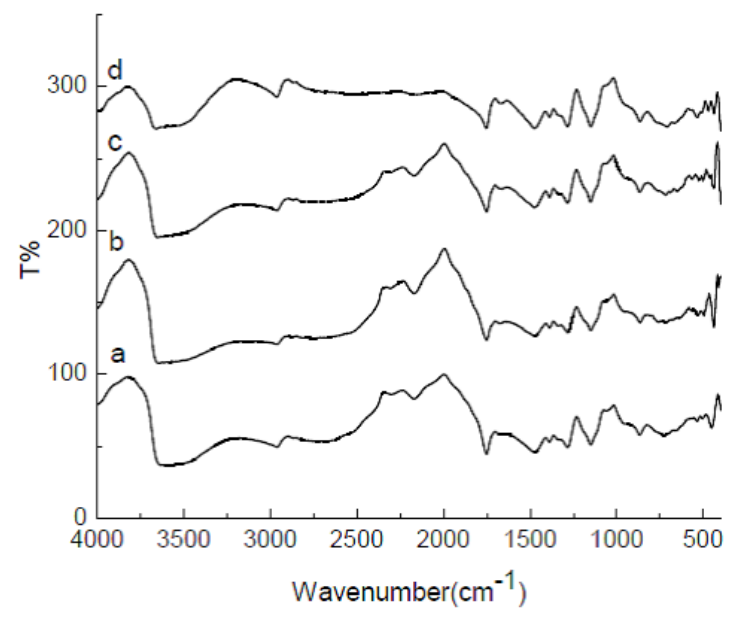

Fig. 6: FTIR spectra of PVA/cur nanocomposites with different concentrations of curcumin (a) $0 \%$, (b) $5 \mathrm{wt} \%$, (c) $10 \mathrm{wt} \%$, and (d) $15 \mathrm{wt} \%$.

The high permittivity of CS/cur and PVA/Cs/cur may be due to the polar acetate and $\mathrm{NH}_{3}^{+}$ions forming during the preparation of films by dissolving chitosan in $2 \%$ acetic acid solution.

The increase in permittivity of the matter could be related to the various forms of polarization. The well-known types of polarizations are electronic polarization, atomic polarization as well as orientation polarization attributed to the orientation of dipoles parallel to the applied electric field (Johns and Charoen, 2011). In addition, in the case of heterogeneous materials, a chance for interfacial polarization to be appears as a result of the distinction in conductivities of the phases. According to the type of polarization, the time needed to reach equilibrium level varies. In our case, which is considered to be a polar molecule, at low frequencies all the four types polarization contribute the permittivity leading to high permittivity, at lower frequencies. By increasing the frequency, both interfacial polarization and the orientation polarization contributions vanished which is the cause of the reduction of the permittivity at higher frequencies. The orientation polarization requires a longer time to reach static field value incomparable with both electronic and atomic polarization. Therefore, the orientation polarization decreases with increase in frequency compared to electronic and atomic polarizations at lower frequency region. At lower frequencies, the surface polarization generally happens at abundant. Anyhow the permittivity $\varepsilon^{\prime}$ was found to follow the order $\mathrm{Cs}>\mathrm{PVA} / \mathrm{Cs} / \mathrm{cur}>$ PVA nanocomposites. 


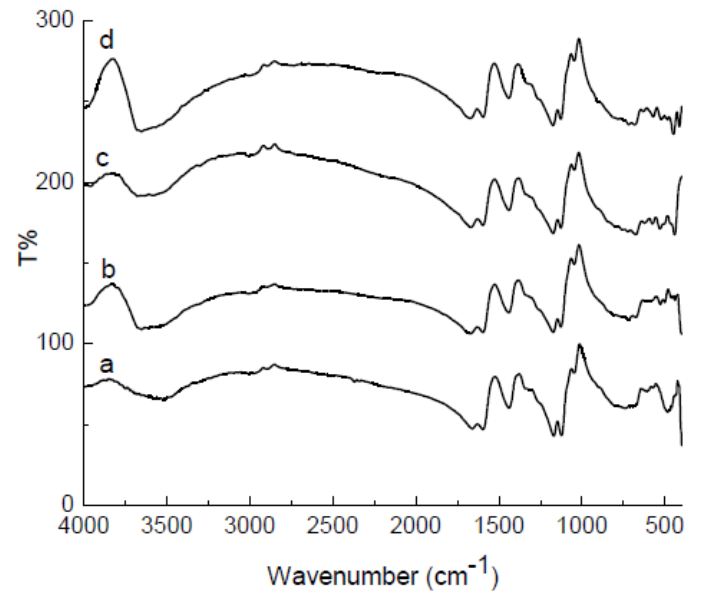

Fig. 7: FTIR spectra of Cs/cur nanocomposites with different concentrations of curcumin (a) $0 \%$, (b) $5 \mathrm{wt} \%$, (c) $10 \mathrm{wt} \%$, and (d) $15 \mathrm{wt} \%$.

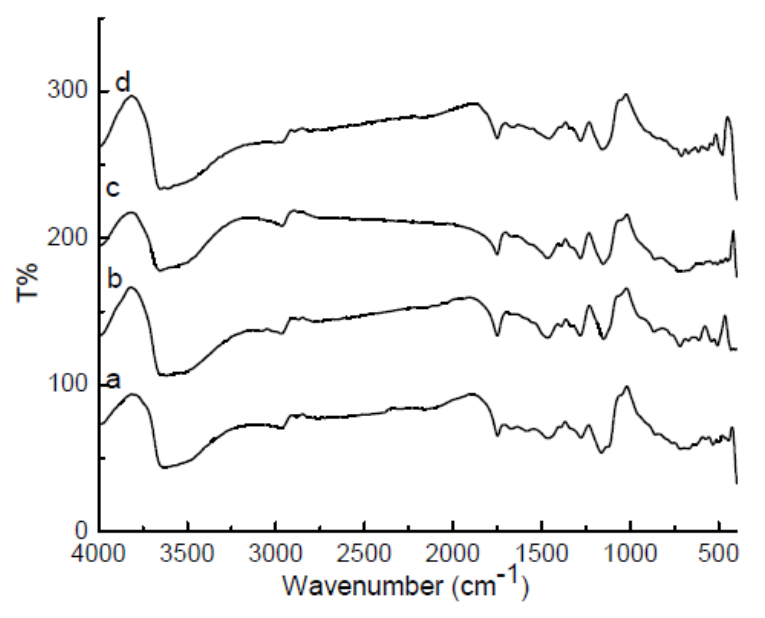

Fig. 8: FTIR spectra of PVA/Cs/cur nanocomposites with different concentrations of cur (a) $0 \%$, (b) $5 \%$, (c) $10 \%$, and (d) $15 \%$.

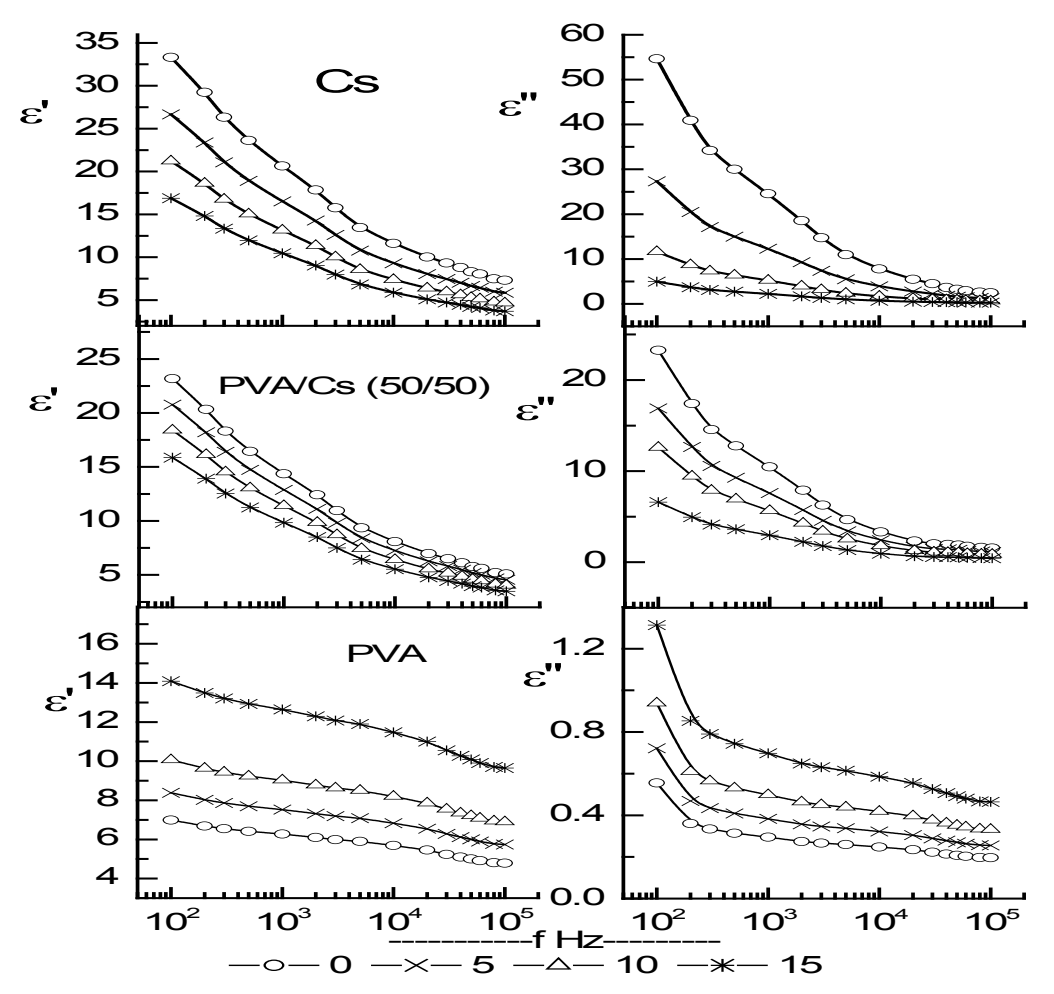

Fig. 9: The permittivity $\varepsilon^{\prime}$ and dielectric loss $\varepsilon^{\prime \prime}$ for cur loaded PVA, Cs and PVA/Cs with different conc. versus the applied frequency f, at room temperature $25^{\circ} \mathrm{C}$.

As a result of the inability of polarization mechanism of the molecules to follow the change of the oscillating of the electric field, the dielectric loss $\varepsilon^{\prime \prime}$ was formed. This type of the relaxation process in a composite is defined as the time taken for the dipoles to come back to its original random orientation. It doesn't take place instantaneously but the polarization tailing exponentially. Minimum loss obtained if the relaxation time is smaller or similar to the rate of the oscillating electric field. On the other hand, the polarization cannot follow the oscillating frequency leading to the energy absorption and or dissipated as heat in case of faster electric field oscillation than the relaxation time (Lovell et al., 1993).
The values of $\varepsilon^{\prime \prime}$ obtained for the nanocomposites under investigation is found to follow the order Cs $>$ PVA/Cs $>$ PVA. Also, it is seen that the values of $\varepsilon^{\prime \prime}$ are higher at low-frequency range beside the fact that $\varepsilon^{\prime \prime}$ versus applied frequency curves are much complicated and broader than one Debye process. This finding reflects more than one relaxation process is expected to happen in such complicated nanocomposites (Kamel et al., 2015; Motawie et al., 2016).

Analyses of these curves were done by using Fröhlich and Havriliak-Nagami functions as mentioned elsewhere (AbdEl-Messieh and Abd-El-Nour 2003). 
Dielectric spectroscopy is a strong tool for studying the dynamics of polymers in an exceedingly wide range frequency. The various regimes of the spectra may be detected and therefore the dynamics of the primary and secondary relaxations were obtained.

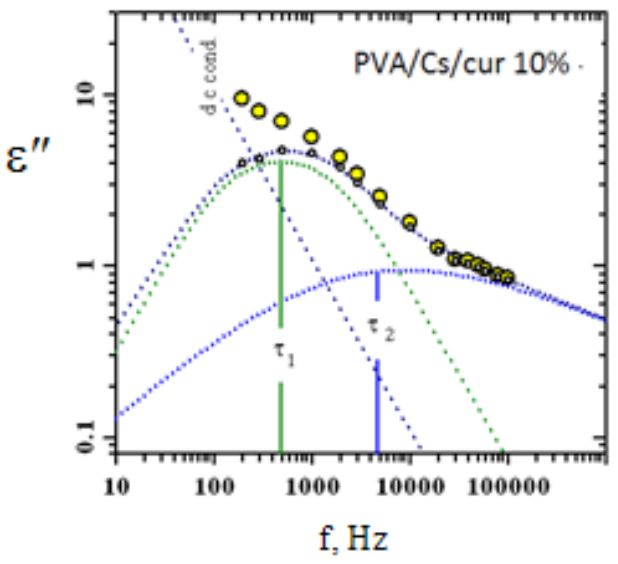

Fig. 10: Example of the analyses of $\varepsilon^{\prime \prime}$ data for $10 \%$ cur loaded PVA/Cs Fitting the experimental data after subtraction of the losses due to dc conductivity with Fröhlich and Havriliak Negami functions.

Example of the analysis is illustrated graphically in Figure 10. From this figure, an absorption region obtained at lower frequency range was fitted by Fröhlich function with

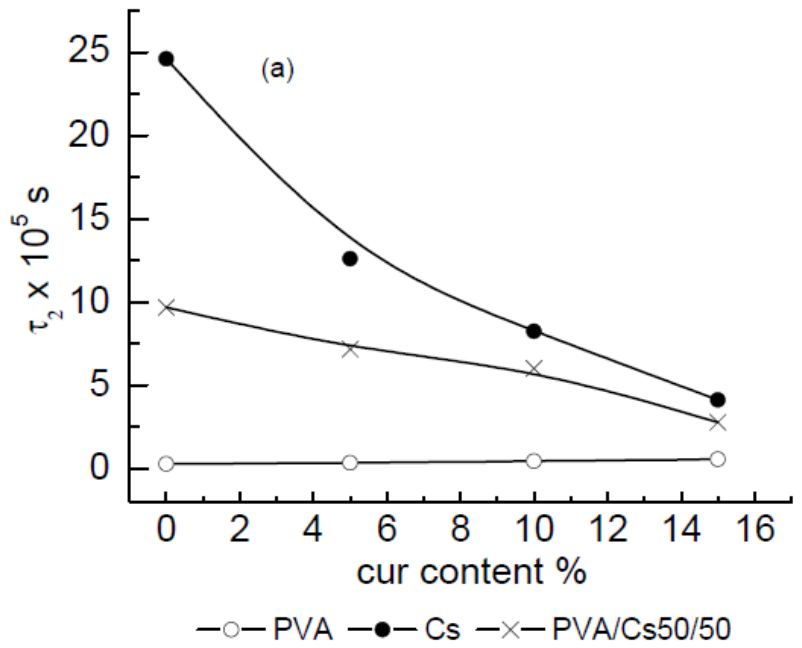

distribution parameter $\mathrm{p}=3$ and relaxation time $\tau_{1}$ about 0.003 $\mathrm{s}$. These values are found to be independent on either the type of polymer or curcumin content in the composite. This region could be attributed to Maxwell-Wagner effect which is expected to be at the lower frequency range as a result of the multi-constituents of the investigated systems. The second absorption region, $\tau_{2}$ which was fitted by Fröhlich distribution could be due to some local molecular motions rather than the main chain motion as it is expected to be frozen since the measurements were carried out at $25^{\circ} \mathrm{C}$ i.e. lower than the glass transition $\mathrm{Tg}$ of the polymers under investigations (Abou-Aiad et al., 2006). The relaxation time $\tau_{2}$ associated with this region is highly affected by the type of polymer and the percentage of curcumin and follows the order Cs $>$ PVA/Cs > PVA.

The obtained data of $\tau_{2}$ for all nanocomposites under investigations are plotted graphically in Figure 11. From this figure, it is clear that $\tau_{2}$ increases in the same order CS $>$ /PVA/ $\mathrm{Cs}>\mathrm{PVA}$ but $\tau_{2}$ values for PVA/Cs composites are much closer to those for Cs rather than PVA indicating a nonlinear relationship. As an example, Figure $11 \mathrm{~b}$ represents the variation of PVA content versus $\tau_{2}$ for PVA/Cs/cur $10 \%$. From this figure, it is clear that the deviation from the line connecting the two individuals was a positive deviation. This finding reflects an interaction between the two polymers which justify the results obtained before from FTIR spectroscopy. This interaction increases the molar volume of the rotating units and then the relaxation time.

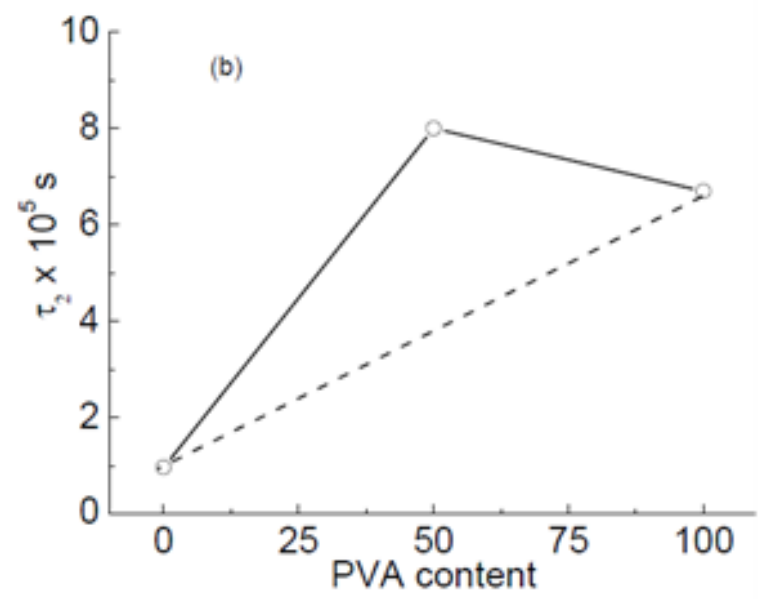

Fig. 11: Relaxation time $\tau_{2}$ versus (a) cur content and (b) PVA content for $10 \%$ cur loaded PVA/Cs.

For evaluation of the insulating materials, the conductivity studies are so important parameter. This is due to the fact that the main objective for an insulator material is to test its capability to oppose the leakage of electric current (Johns and Charoen, 2011). The electrical conductivity $\sigma$ was calculated from measured resistance and illustrated graphically in Figure 12. From this figure, it is found that $\mathrm{Cs} /$ cur nanocomposites possess the highest $\sigma$ values while PVA/cur are the lowest ones. As seen in figure 12 the $\sigma$ values are found to decrease by increasing curcumin content in both $\mathrm{C}_{\mathrm{s}}$ and PVA/CS nanocomposites. While on the other hand, they increase by increasing cur content in PVA/ cur nanocomposites. Anyhow, the electrical conductivity of both Cs (Martins et al., 2014) and PVA (El-Houssiny et al., 2012) are found to be comparable with those found in the literature.

However, the obtained electrical conductivity for all nanocomposites under investigation lies in the order of the antistatic range $10^{-9}-10^{-14} / \mathrm{S} \mathrm{cm}^{-1}$ (Huang, 2002) which recommend being used for antistatic applications such as skin contact.

\section{Water vapor transmission rate (WVTR)}

WVTR is the ability of a dressing material to control water loss from the wound. Moderate WVTR is recommended to 
offer a moist environment to accelerate healing. High WVTR may cause dryness of the wound, while low WVTR may cause storing of wound exudates which may result in retardation of the wound healing (Xu et al., 2016). The values of the WVTR for normal skin have been reported to be in the range $8.5 \mathrm{~g} / \mathrm{m}^{2} / \mathrm{h}$ to $11.6 \mathrm{~g} /$ $\mathrm{m}^{2} / \mathrm{h}$ (Gonzalez et al., 2012). The WVTR values for cur loaded PVA, Cs and PVA/Cs blend are shown in Table 2. From this table, it is obvious that addition of cur decreases the WVTR of the composites. WVTR values follow the order PVA $>$ Cs $>$ PVA/Cs.

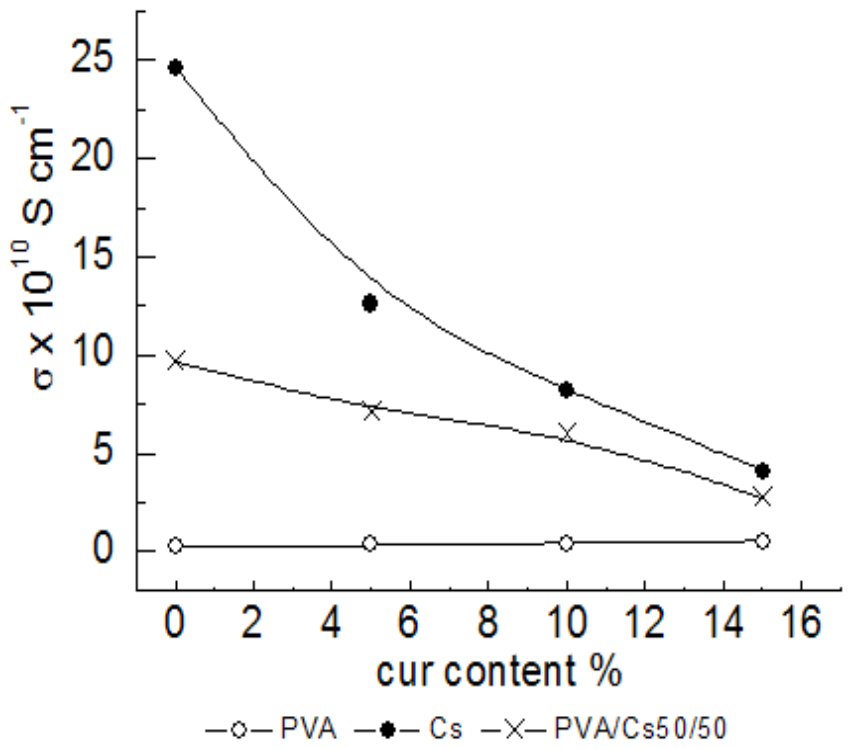

Fig. 12: Electrical de conductivity versus cur content.

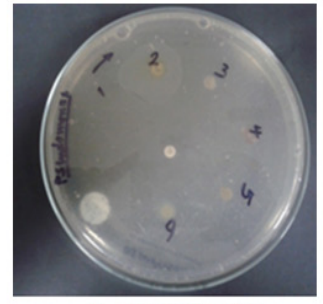

P. aeruginosa

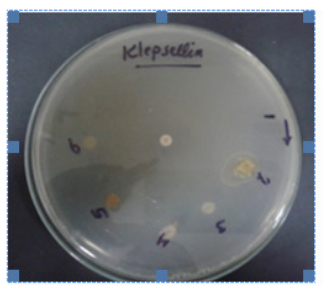

K. pneumonia

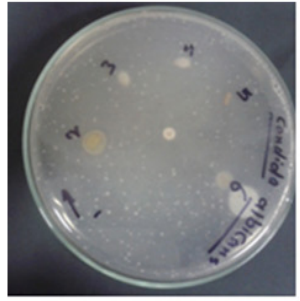

Can. Albicans

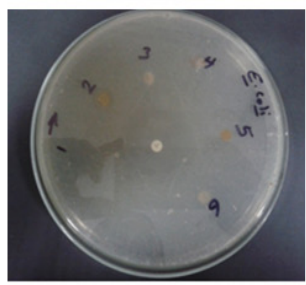

E.coli

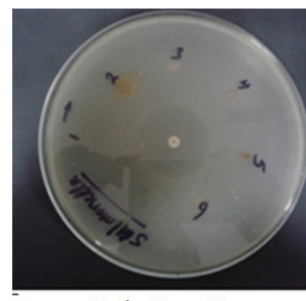

Sal. Enterica

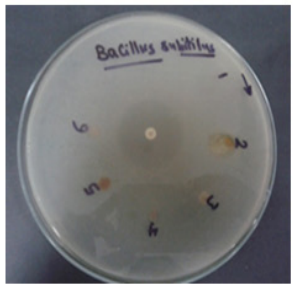

B. subtilis

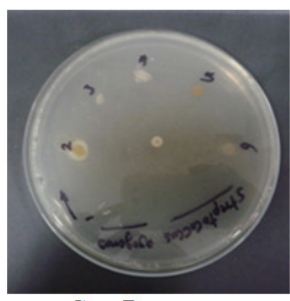

Str. Pyogenes

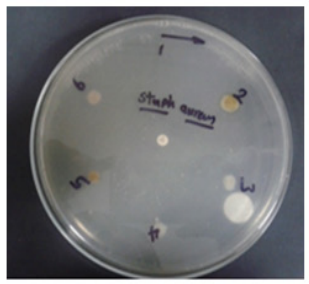

Staph. Aureus

Fig. 13: Zone of inhibition of 0 and $10 \%$ loaded cur PVA, Cs and PVA/Cs, PVA/Cs blend against various pathogenic microorganisms.

\section{Cell viability assay}

The cell viability effect of pure and $10 \%$ cur loaded PVA, $\mathrm{Cs}$ and PVA/Cs blend against the human normal melanocyte cell line (HFB4) was investigated and presented in Table 3. Cultures of the cell line were treated with extracts first at one concentration of $100 \mu \mathrm{g} / \mathrm{ml}$ and the results indicated the all samples showed cell viability greater than $80 \%$ which confirm that addition of cur has no toxic effect on the cells.

\section{Antimicrobial assay}

Figure 13 represents the Zone of inhibition of pure and $10 \%$ cur loaded nanocomposites against 8 pathogenic microorganisms and the Inhibition zone diameter (millimetre) of the pure and $10 \%$ cur loaded PVA, Cs and PVA/Cs nanocomposites is represented in Table 4. The results illustrated that the samples PVA, Cs, Cs/cur 10\% have very weak antimicrobial effect on all tested standard microorganisms, while the sample PVA/Cs/ 
cur $10 \%$ has moderate antimicrobial activity showed on some gram negative bacteria (Klebsiella pneumonia) \& gram positive bacteria (Streptococcus pyogenes) with inhibition zone diameter (11 mm), also the PVA/Cs blend showed low to moderate on all tested bacterial either gram positive or gram negative bacteria with inhibition zone diameter ranged (7-14 mm). On the other hand there is no antimicrobial effect on yeasts (Candida albicans) by all tested samples except the sample PVA/cur $10 \%$ which showed moderate antimicrobial effect with inhibition zone diameter $(19 \mathrm{~mm})$, moreover, the sample PVA/cur $10 \%$ was the best one which showed moderate to strong antimicrobial effect on all tested standard microorganisms especially on Klebsiella pneumonia which is pathogen that mainly involved in many infections and can be linked with a high rate of mortality. Previous work on curcumin confirmed its efficiency against this pathogen (Gunes et al., 2016).

When chitosan and PVA/Cs blend are loaded with curcumin, they have chemically interacted as supposed by FTIR and dielectric results. This interaction may lead to limit the inhibitory effect of curcumin as observed in the antimicrobial test. On the other hand no or few interactions between PVA and curcumin so the active compounds of curcumin are free to inhibit microorganisms in the antimicrobial test and this may be the interpretation of the increased antimicrobial efficiency of the $\mathrm{PVA} /$ cur than cs/cur nanocomposites.

Table 2: Water vapor transmission values for cur loaded PVA, Cs and PVA/Cs blend.

\begin{tabular}{ccccc}
\hline \multirow{2}{*}{ Sample } & \multicolumn{4}{c}{ WVTR values $\mathbf{g} / \mathbf{m}^{2} / \mathbf{h}$} \\
\cline { 2 - 5 } & $\mathbf{0 \%}$ cur & $\mathbf{5 \%}$ cur & $\mathbf{1 0 \%}$ cur & $\mathbf{1 5 \%}$ cur \\
\hline PVA & 37.81 & 35.82 & 30.78 & 24.42 \\
Cs & 19.90 & 17.78 & 16.80 & 16.72 \\
PVA/Cs blend & 11.94 & 9.29 & 9.28 & 8.625 \\
\hline
\end{tabular}

Table 3: Cell viability of pure and $10 \mathrm{wt} \%$ cur loaded PVA, Cs and PVA/Cs blend $(100 \mu \mathrm{g} / \mathrm{ml})$ on human normal melanocyte cell line (HFB4).

\begin{tabular}{cc}
\hline \multirow{2}{*}{ Sample } & Cell viability\% \\
\cline { 2 - 2 } & HFB4 (human Normal melanocyte) \\
\hline PVA & 91.3 \\
PVA/cur $10 \%$ & 88.6 \\
Cs & 91 \\
Cs/cur $10 \%$ & 91.1 \\
PVA/Cs & 84.1 \\
PVA/Cs/cur $10 \%$ & 83.5 \\
\hline
\end{tabular}

Table 4: Inhibition zone diameter (millimeter) of the pure and 10\% cur loaded PVA, Cs and PVA/Cs nanocomposites.

\begin{tabular}{|c|c|c|c|c|c|c|c|}
\hline \multirow{2}{*}{ Microorganism } & 1 & 2 & 3 & 4 & 5 & 6 & \multirow{2}{*}{ LEV.** } \\
\hline & PVA & PVA/cur & Cs & Cs/cur & PVA/Cs & PVA/Cs/cur & \\
\hline E. coli & $\mathrm{Nil}^{*}$ & 12.0 & Nil & Nil & 8.0 & Nil & 24.0 \\
\hline Sal. enterica & Nil & 24.0 & Nil & Nil & 10.0 & Nil & 38.0 \\
\hline K. neumoniae & Nil & 26.0 & Nil & Nil & 9.0 & 11.0 & 27.0 \\
\hline P. aeruginosa & Nil & 18.0 & Nil & Nil & 14.0 & Nil & 30.0 \\
\hline Str. pyogenes & Nil & 20.0 & Nil & Nil & 11.0 & 11.0 & 24.0 \\
\hline Staph. aureus & Nil & 21.0 & Nil & Nil & 9.0 & Nil & 26.0 \\
\hline B. subtilis & Nil & 15.0 & Nil & Nil & 7.0 & Nil & 36.0 \\
\hline Can. albicans & Nil & 15.0 & Nil & Nil & Nil & Nil & 32.0 \\
\hline
\end{tabular}

*Nil: No antimicrobial activity recorded.

**LEV: Levofloxacin, used as a standard antibiotic.

\section{CONCLUSION}

PVA, chitosan and their 50:50 blend nanocomposite with and without curcumin have been prepared by solution casting method using different concentrations of curcumin $(0,5,10$ and 15) $\mathrm{wt} \%$. Results of the FTIR suggest an interaction between the nanocurcumin and chitosan and also between the curcumin and the PVA/Cs blend. Results of the SEM indicate good dispersion of the curcumin within the polymers matrix. Cur loaded PVA have the highest WVTR than chitosan and PVA/Cs blend. The Low WVTR induced by cur loading suggests that these types of dressings are more suitable for nonexudative or dry wounds. The cell viability data indicated that all samples showed cell viability greater than $80 \%$ which confirmed that the addition of curcumin has no cytotoxic effect on human normal melanocytes. PVA/cur nanocomposites are the best which showed moderate to strong antimicrobial effect on all tested standard microorganisms. Both $\varepsilon^{\prime}$ and $\varepsilon^{\prime \prime}$ values follow the order $\mathrm{CS}>/ \mathrm{PVA} / \mathrm{Cs}>\mathrm{PVA}$. The obtained electrical conductivity for all composites under investigation lies in the order of the antistatic range $\left(10^{-9}-10^{-14} / \mathrm{S} \mathrm{cm}^{-1}\right)$ which recommend such composites to be used for antistatic applications. The investigation indicates that the PVA/cur nanocomposites have high potential as wound dressing materials and in-vivo study for these materials are recommended.

\section{REFERENCES}

Abd-El-Messieh SL, Abd-El-Nour KN. Effect of curing time and sulfur content on the dielectric relaxation of styrene butadiene rubber. J App Poly Sci. 2003; 7:1613-1621.

Abou-Aiad THM, Abd-El-Nour KN, Hakim IK, Elsabee MZ. Dielectric and interaction behavior of chitosan/polyvinyl alcohol and chitosan/polyvinyl pyrrolidone blends with some antimicrobial activities. Poly. 2006; 47:379-89.

Aggarwal BB, Kuzhuvelil BH. Potential therapeutic effects of curcumin, the anti-inflammatory agent, against neurodegenerative, cardiovascular, pulmonary, metabolic, autoimmune and neoplastic diseases. 
Intl J Biochem Cell Biol. 2009; 41:40-59.

Baker MI, Walsh SP, Schwartz Z, Boyan BD. A review of polyvinyl alcohol and its uses in cartilage and orthopedic applications. J Biomed Mater Res B Appl Biomater. 2012; 100:1451-1457.

Chen Y, Lin J, Fei Y, Wang H, Gao W. Preparation and characterization of electrospinning PLA/curcumin composite membranes. Fibers Poly. 2010; 11:1128-1131.

Chuang WY, Young TH, Yao CH, Chiu WY. Properties of the poly (vinyl alcohol)/chitosan blend and its effect on the culture of fibroblast in vitro. Biomaterials. 1999; 20:1479-1487.

Das RK, Naresh K, Utpal B. Encapsulation of curcumin in alginate-chitosan-pluronic composite nanoparticles for delivery to cancer cells. Nanomedicine: Nanotechnology, Biology and Medicine. 2010; 6:153160 .

Dash M, Chiellini F, Ottenbrite RM, Chiellini E. Chitosan - A versatile semi-synthetic polymer in biomedical applications. Prog poly sci. 2011; 36:981-1014.

Duvoix A, Blasius R, Delhalle S, Schnekenburger M, Morceau F, Henry E, Dicato M, Diederich M. Chemopreventive and therapeutic effects of curcumin. Cancer Lett. 2005, 223:181-190.

El-Hefian, Esam A, Mohamed MN, Abdul Hamid Y. The preparation and characterization of chitosan/poly (vinyl alcohol) blended films. J Chem. 2010; 7:1212-1219.

El-Houssiny AS, Ward AAM, Mansour SH, Abd-El-Messieh SL. Biodegradable blends based on polyvinyl pyrrolidone for insulation purposes. J App Poly Sci. 2012; 124:3879-3891.

El-Nashar DE, Rozik NN, Soliman M, Helaly F. Study the release kinetics of curcumin released from $\mathrm{PVA} /$ curcumin composites and its evaluation towards hepatocarcinoma. J App Pharm Sci. 2016; 6:067-072.

Fan X, Zhang C, Liu D, Yan J, Liang HP. The clinical applications of curcumin: current state and the future. Curr Pharm Des. 2013; 19:20112031.

Gonzalez JS, Hoppe CE, Alvarez VA, Maiolo S. Composite gels based on poly (vinyl alcohol) for biomedical uses. Procedia Mate Sci. 2012; 1:483-490.

Gunes H, Gulen D, Mutlu R, Gumus A, Tas T, Topkaya AE. Antibacterial effects of curcumin: an in vitro minimum inhibitory concentration study. Toxicol Ind Health. 2016; 32:246-250.

Huang JC. Carbon black filled conducting polymers and polymer blends. Advances in Poly Tech. 2002. 21:299-313.

Andrews JM. Determination of minimum inhibitory concentrations. J Antimicrob Chemother. 2002; 49:1049.

Johns J, Charoen N. Dielectric properties of natural rubber/ chitosan blends: Effects of blend ratio and compatibilization. J of NonCrystalline Solids. 2011; 357:1816-1821.

Kamel NA, Mansour SH, Abd-El-Messieh SL, Khalil WA. AbdEl Nour KN. Biophysical properties of PPF/HA nanocomposites reinforced with natural bone powder. Adv mat Res. 2015; 4:145-164.

Kamel NA, Abd-El-Messieh SL, Neveen MS. Chitosan/banana peel powder nanocomposites for wound dressing application: Preparation and characterization. Materials Sci Eng. 2017; 72:543-550.

Khan MS. Electrical and optical properties of some oils seeds and grains. Thesis. 2014

Lovell PA, McDonald J, Saunders DEJ, Young RJ. Studies of rubber-toughened poly (methyl methacrylate): 1. Preparation and thermal properties of blends of poly (methyl methacrylate) with multiple-layer toughening particles. Polym. 1993; 34:61-69.

Martins AM, Eng G, Caridade SG, Mano JF, Reis RL, VunjakNovakovic G. Electrically conductive chitosan/carbon scaffolds for cardiac tissue engineering. Biomac. 2014; 15:635-643.

Mosmann T. Rapid colorimetric assay for cellular growth and survival: application to proliferation and cytotoxicity assays. J Immuno Methods. 1983; 65:55-63.

Motawie AM, Mansour NA, Kandile NG, Abd-El-Messieh SL, El-Mesallamy SM, Sadek EM. Study on the Properties of Carbon Reinforced Unsaturated Thermoset Polyester Resin Nanocomposites. Aust. J. Basic \& Appl. Sci. 2016; 1:37-47.

Nakano Y, Bin Y, Bando M, Nakashima T, Okuno T, Kurosu $\mathrm{H}$, Matsuo M. Structure and mechanical properties of chitosan/poly (vinyl alcohol) blend films. Macromol Symp. 2007; 258:63-81.

Phan TT, See P, Lee ST, Chan SY. Protective effects of curcumin against oxidative damage on skin cells in vitro: its implication for wound healing. J Trauma Acute Care Surg. 2001; 51:927-931.

Priyadarsini KI. The chemistry of curcumin: from extraction to therapeutic agent. Molecules. 2014; 19:20091-20112.

Rahman MU, Shereen G, Zhoor UL HM. Reduction of chromium (VI) by locally isolated Pseudomonas sp. C-171. Turk J Bio. 2007; 31:161166.

Razzak MT, Darwis D. Irradiation of poly (vinyl alcohol) and poly (vinyl pyrrolidone) blended hydrogel for wound dressing. Radiat Phys Chem. 2001; 62:107-113.

Shankar S, Rakesh KS. Curcumin: structure, biology and clinical applications. Nutrition, Diet and Cancer. 2012; 413-457.

Srivastava M, Singh J, Mishra R, Sudhanshu S. Novel conducting lithium ferrite/chitosan nanocomposite: Synthesis, characterization, magnetic and dielectric properties. Curr Appl Phys. 2014; 14:980-990.

Tang H, Murphy CJ, Zhang B, Shen Y, Van Kirk EA, Murdoch WJ, Radosz M. Curcumin polymers as anticancer conjugates. Biomat. 2010; 31:7139-7149.

Varaprasad K, Mohan Y M, Vimala K, Mohana KR. Synthesis and characterization of hydrogel-silver nanoparticle-curcumin composites for wound dressing and antibacterial application. J App Poly Sci. 2011; 121:784-796

Vidyalakshmi K, Rashmi KN, Kumar TMP, Siddaramaiah. Studies on formulation and in vitro evaluation of $\mathrm{PVA} /$ chitosan blend films for drug delivery. J Macromol Sci, Part A. 2004; 41:1115-1122.

Wang T, Mahir T, Sundaram G. Selected properties of $\mathrm{pH}$ sensitive, biodegradable chitosan-poly(vinyl alcohol) hydrogel. Poly Int 2004; 53:911-918.

Weber WM, Hunsaker LA, Abcouwer SF, Deck LM, Vander Jagt DL. Anti-oxidant activities of curcumin and related enones. Bioorganic Med Chem. 2005; 13:3811-3820.

Xu R, Xia H, He W, Li Z, Zhao J, Liu B, Wang Y, Lei Q, Kong Y, Bai Y, Yao Z, Yan R, Li H, Zhan R, Yang S, Luo G, Wu J. Controlled water vapor transmission rate promotes wound-healing via wound reepithelialization and contraction enhancement. Sci Rep. 2016; 6.

Zhao Z, Xie M, Li Y, Chen A, Li G, Zhang J, Hu H, Wang X, Li S. Formation of curcumin nanoparticles via solution-enhanced dispersion by supercritical $\mathrm{CO}_{2}$. Int J Nanomedicine. 2015; 10:3171.

How to cite this article:

Kamel NA, Soliman AAF, Rozik NN, Abd-Elmessieh SL. Biophysical investigation of curcumin based nanocomposite for wound dressing application. J App Pharm Sci, 2018; 8(05): 035044. 